\title{
Prevalence of Tuberculosis in Mediterranean Port City Tobruk, Libya: A Retrospective Study
}

\author{
Faisal Ismail ${ }^{1,2}$, Atiya Farag', ABDUL Ilah ${ }^{1}$ \\ ${ }^{1}$ Department of Medical Laboratory, Faculty of Medical Technology, University of Tobruk, Tobruk, Libya \\ ${ }^{2}$ National Centre for Disease Control, Tobruk, Libya
}

\begin{abstract}
High prevalence and high rate of transmission of infection is the characteristic feature of the tuberculosis (TB). The pre and post revolution situation of Tobruk in Libya has been closely studied in the National Center for TB \& Chest Diseases. We have presented here identified TB cases of about 40 years since 1974 till 2014 based on extensive smear/ culture positive examination of the samples. The study included 995 cases out of that $60.4 \%$ male and $39.6 \%$ female cases. The majority of cases $582(85.6 \%)$ were Libyans and 14.4\% non-Libyan nationalities, where Egyptian were, 46 (4.6\%), Sudanese 15 (1.5\%), and small percentages were from other nationalities. The trend of tuberculosis infection had been found to be statistically very uneven during four decades among different types of patients group such as male, female, young, old and with different occupations and nationalities. The highest infection was recorded in 1976 (total cases 64) and lowest in 1999 (total cases 5). The cases increased gradually and found to vary between 7 to 33 cases almost every year. The identified 390 cases (39.2\%) were Pulmonary TB cases and the remaining 605 cases (60.8\%) were extrapulmonary TB cases. Nearly half of the TB cases (49.7\%) were among the age group 15 to 34 years. The highest number of cases was from Tobruk city 859 cases (86.3 \%), followed by Emsaed city on the Libyan Egyptian border 44 cases (4.4\%). Thus we reported here that Tobruk is the middle burden city for tuberculosis within Libya.
\end{abstract}

Keywords: Tobruk, Tuberculosis, Age, Gender, Occupation

\section{Introduction}

Tuberculosis (TB) has been one of the major challenges for whole humanity for its burgeoning morbidity and mortality worldwide, especially in the developing countries. According to World health organization (WHO) around 8.8 million new cases arises and 1.1 million patients died from TB in the year of 2010. The frequency of the disease were found to be more in Asia (59\%) and Africa (26\%) [1]. The 40 cases per 100.000 populations of TB cases in whole Libya estimated in the period of 2010 and the general frequency was 53 cases per 100.000 populations [2].

Despite the fact that the estimated number of people gets infected with TB infection each year is declining, TB infection continues to be a major public health problem in the world; the World Health Organization (WHO) estimated that 9 million people with $\mathrm{TB}$ infection in 2013, and 1.5 million died from the disease[3].

In Libya several studies were performed to evaluate the incidence of TB in different regions of Libya; long back one study was performed in some regions of Eastern Libya found that the incidence of TB infection is 1.19-2.47\% in 1959 [4]. Similar study was performed in 1977 included 51,114 people and showed an incidence of $33 / 100,000$ of population [5]. In recent past a study was performed to estimate the incidence of pulmonary Tuberculosis in North western Libya included 1559 cases. Out of these $51.0 \%$ cases were pulmonary TB cases; all data of this pulmonary TB cases since 2003 were collected from the annual notification reports of the National Centre of Tuberculosis \& Chest diseases monitoring in Tripoli city [6]

Tobruk city is the capital of Butnan which is located on the Libya's eastern Mediterranean coast, with a population of 160,000 (2006), with a $140 \mathrm{~km}$ long on the Mediterranean, it has international border with Egypt in the east and district of Derna located northwest and Al Wahat district in the south west[7].

In fact that there were no comprehensive surveys conducted in Tobruk area so far, this study was conducted to estimate the health burden of tuberculosis in Tobruk region and aiming at to get a comprehensive record of incidence of tuberculosis in this region since last four decades among Libyans and non- Libyans. The research based survey was conducted by covering wide parameter including, pulmonary and extra pulmonary tuberculosis registered in the National Centre for Tuberculosis \& Chest diseases monitoring in Tobruk. In addition we also cover in our study the Gender, Age groups, Nationalities and Occupations affected by tuberculosis and the geographic distribution of the cases.

\section{Materials and Methods}

\section{Study Location}

The study was conducted at National Center for TB \& Chest Diseases situated in Tobruk. Tobruk is the district port city of State Butnan of Libya, situated $1500 \mathrm{~km}$ away from east of capital Tripoli. The National Center of TB \& Chest Diseases is serving at least 160000 population of Tobruk and the surrounding remote areas of the city since long. The National Center of TB \& Chest Diseases is operating under the National Tuberculosis Control Program, under which patients are diagnosed with tuberculosis by examination of morning sputum smears by Zeihel-Nieelsen staining, for the presence of Acid fast bacilli (AFB), chest radiographs for extra pulmonary tuberculosis (EPTB), pathological investigations were used. Patients were registered and treated according to the National Tuberculosis Control Program of Libya.

Study design and data collection 


\section{International Journal of Science and Research (IJSR) \\ ISSN (Online): 2319-7064}

Index Copernicus Value (2013): 6.14 | Impact Factor (2014): 5.611

A forty- years retrospective descriptive analysis to assess the Tuberculosis cases and risk factors of 995 TB patients, registered from 1974 to 2014 was carried out in National Center for TB \& Chest Diseases. All TB patients registered at the National Center for TB \& Chest Diseases were followed up during their course of treatment for complete assessment of TB cases. Demographic data such as patient's age, sex, nationality, occupations as well as the tuberculosis type were included in the registration form. Patients were followed up regularly until completion of their treatment.

\section{Statistical analysis}

Data were analyzed using the Statistical Package for the Social Sciences (SPSS) software package, Version 19. Data was analyzed using SPSS computer software (Version 19, SPSS Inc.). The TB cases were divided into groups and the different variables in the study such as age, gender and the geographic distribution of the cases were examined and compared to previous studies from Libya and some regional $\&$ international countries.

\section{Results}

Age intervals distribution in TB cases: Ages of patients were divided into age groups (Figure1). The most TB cases were found to be among age group 15 to 34 years (49.7\%) out of 995 cases. The 390 patients $(39.2 \%)$ were from pulmonary TB cases and the remaining 605 cases $(60.8 \%)$ were from extra-pulmonary TB cases. (Figure 1) Gender distribution in TB cases: The gender in TB cases was taken into consideration. Among all (995) registered cases from 1974 to 2014, domination of diseases were among male $60.4 \%$ and less tendency of occurring TB cases among female i.e $39.9 \%$. (Figure 2)

\section{Different nationality Distribution in TB cases:}

The several nationalities who were occupying the Tobruk region along with Libyan were found to be infected with tuberculosis bacillus. It is statistically revealed that $85.6 \%$ were Libyans who got TB infections during study period. The rest $14.4 \%$ TB patients were non-Libyan nationalities. Other than Libyan nationalities, Egyptians were more in number who got infection (4.6\%), followed by Sudanese TB infections $(1.5 \%)$. Many more nationals were also infected but their infection rate was very less. (Figure 3)

\section{Occupational distribution in TB cases:}

The tuberculosis infections among different occupational category were also statistically evaluated. The most affected category by tuberculosis is found to be housewives followed by self-employed and students. (Figure 4)

\section{Incidence of TB cases over the study period:}

Most of the tuberculosis cases were from Tobruk city (859, $86.3 \%)$ in which the largest percentage of population is inhabited followed by Emsaed town situated near (44 cases $4.4 \%)$ Libyan- Egyptian border. The incidence of tuberculosis in Tobruk area in the past four decades fluctuated between 64 to 20 cases during 1976 to 2014. The highest cases were recorded in 1976. The lowest infection rate was registered in 1987, 1989, 1990, 1991, 1992 and numbers of cases were $7,5,7,7,9$ respectively and no cases registered in year 1988. (Figure 5)

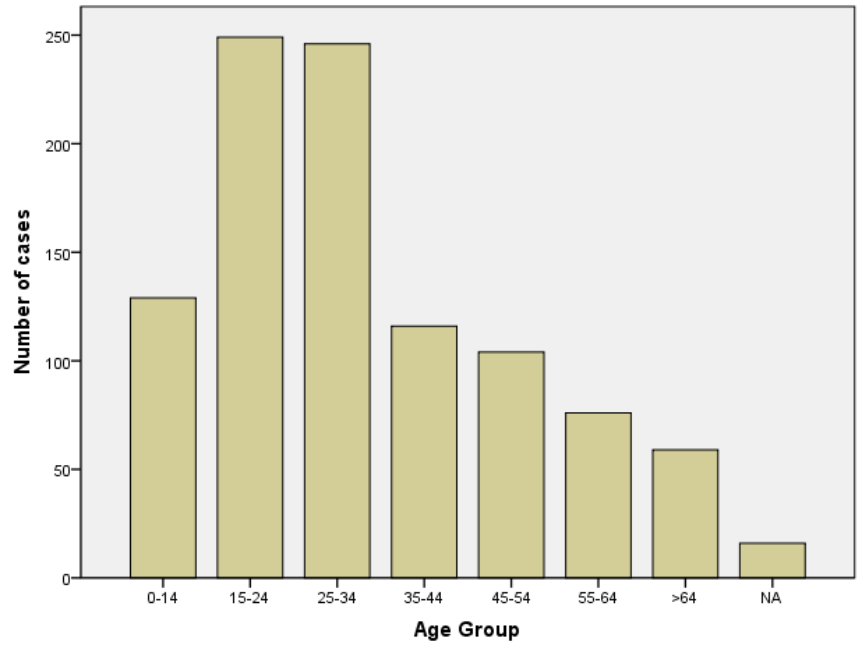

Figure 1: Age intervals distribution of $\mathrm{TB}$ cases (NA= age not available)

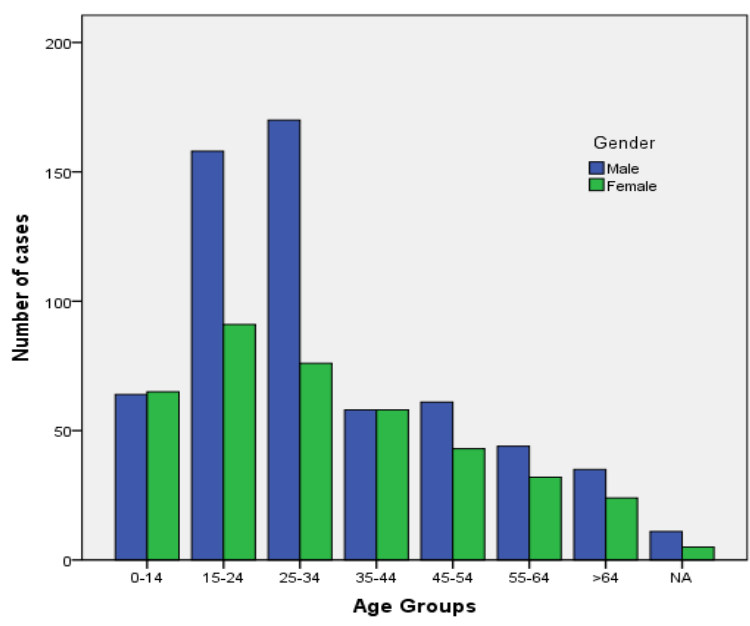

Figure2: Gender distribution of $\mathrm{TB}$ cases $(\mathrm{NA}=$ gender not available)

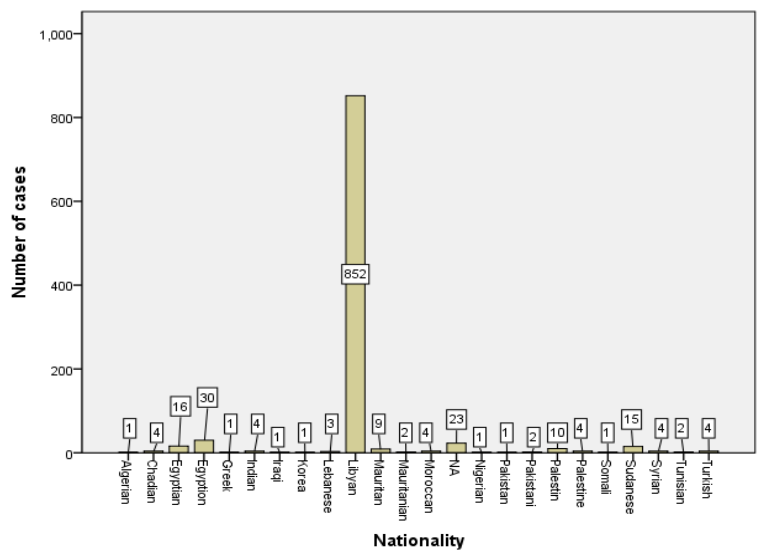

Figure 3: Distribution of TB cases on different nationality $(\mathrm{NA}=$ nationality not available $)$ 


\section{International Journal of Science and Research (IJSR) \\ ISSN (Online): 2319-7064}

Index Copernicus Value (2013): 6.14 | Impact Factor (2014): 5.611

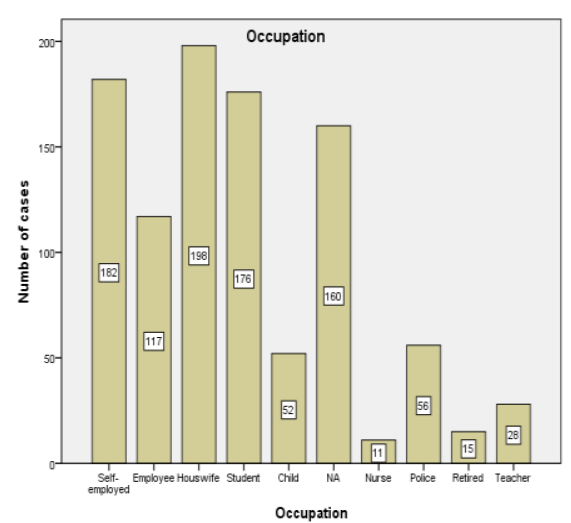

Figure 4: Distribution of TB cases and occupation (NA= occupation not available)

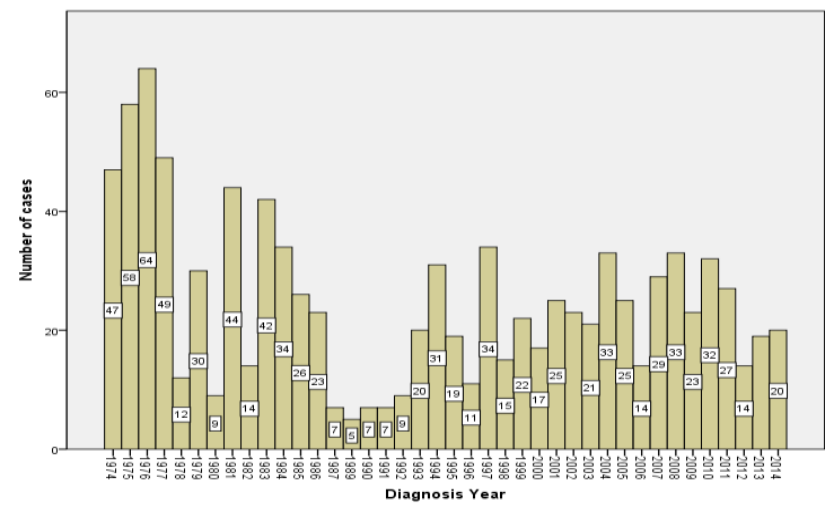

Figure 5: Incidence of TB cases over the study period

\section{Discussion}

Tuberculosis (TB) is one of the principal causes of death in the world. It is reported that globally around 8.8 million people develop tuberculosis and 1.45 million people die every year due to this disease and 0.35 million deaths are associated with HIV- TB co-infection [8]. Mostly increased occurrence of tuberculosis is found to be in Africa and Asia, where the highest incidence of co-infection with HIV and M. tuberculosis occurs . [9-10]. The global burden of death and disease due to $\mathrm{TB}$ is basically centered in low-income countries. The sub-Saharan Africa is the area of highest prevalence of TB infection and A WHO report confirmed that Ethiopia ranks seventh among the world's 22 countries with a high tuberculosis burden [11]. In another report, World health Organization classified Libya as low burden country like many other countries including Jordan, Lebanon, Oman, Tunisia, United Arab Emirates, and Palestine's Gaza Strip \& West Bank (estimated incidence of TB cases were identified between 0-24 TB/100,000 populations).[12] Consistence with the previous study, the average annual incidence of TB cases in our study 25 cases per year has been identified. Previously TB National Centre in Tripoli reported that among all identified TB cases 50.9\% were pulmonary type TB cases and not associated with HIV in Libya similarly in our recent study also confirmed that the identified TB cases were pulmonary and not associated with HIV infections with somewhat lower frequency rate i.e. 39.2 $\%$. Approximately $(85 \%)$ of cases occurs among socially \& economically deprived community and among age groups of 15-34. The infections are found to be dominated among male. Abunaaja et al. [13] also confirmed the same tendency of infection with male. Among the total identified tuberculosis patients about $60.4 \%$ were male slightly higher than women [14-15].

The incidence of TB cases is particularly high in Emsaed town on the Libyan-Egyptian border as WHO classified Egypt among the Middle Burden countries (estimated incidence of 25-49 TB patients/ 100,000 population): together with Bahrain, Iran, Kuwait, Saudi Arabia \& Syria. [16]. The frequent illegal emigration of poor labor class citizens of Egypt to Emsaed town of Libya has probably made this region most vulnerable for these infections.

\section{Conclusion}

A research based comprehensive survey on incidences of Tuberculosis has been conducted in Tobruk region. In this study we have verified incidences and distribution of tuberculosis among gender, age group, occupations and nationalities over time. The tuberculosis cases specially in Tobruk region during last four decades has been closely observed by verifying record collected from National Center of TB \& Chest Diseases. It was observed that $39.2 \%$ patients were suffering from pulmonary TB cases among the total number of verified TB patients during the study period, in which Libyan nationalities were in the majority i.e $85.6 \%$, whereas, non-Libyan cases represented $14.4 \%$,. The most frequent nationalities among non-Libyan cases were from neighboring countries Egypt $4.6 \%$ and Sudanese $1.5 \%$. Most TB cases were among young people age group 15 to 34 years $49.7 \%$ of all cases with predominance of the male sex $60.4 \%$. The incidence of tuberculosis has been found to be fluctuated. In 1976 the tuberculosis cases was highest. Tuberculosis incidence over the period represented an average of 25 cases per year. Our study confirmed that Tobruk is a middle burden city within low burden state of Libya for tuberculosis perhaps this city is very close to Egypt and Sudan both are identified middle burden country for Tuberculosis.

In Libya, compulsory screening measures for all foreignborne workers focused on the identification of active tuberculosis cases should be strongly implemented. Many countries all over the world with a low incidence of tuberculosis use pre-entry screening of immigrants to detect the disease $[17,18,19,20]$

We recommends here for further study to determine the risk factors, which led to the development of TB among the young male population and other groups as well.

\section{Acknowledgement}

The authors would like to acknowledge doctors and health workers at the National Center of TB \& Chest Diseases, Tobruk, Libya

\section{References}

[1] World Health Organization (2011).Global tuberculosis control. WHO report. Geneva, World Health Organization. (http://www.who.int/tb/data). Albarouni 


\section{International Journal of Science and Research (IJSR) \\ ISSN (Online): 2319-7064}

Index Copernicus Value (2013): 6.14 | Impact Factor (2014): 5.611

[2] Awatef A. Ben Ramadan, Mohamed K. Shambesh, Omar I. Abusnena, Mohammad Al-Baccush. Ibnosina: Estimation of the Incidence of Pulmonary Tuberculosis in Northwestern Libya J Med BS, 2011,3(2):53-58

[3] World Health Organization Fact sheet: (2015). N 104 available

from:http://www.who.int/mediacentre/factsheets/fs104/e $\mathrm{n} /$

[4] Kadiki O, Ashraf M. Tuberculosis in the Libyan Arab Republic. Published by Ministry of health (Endemic diseases department): Tripoli Libya 1972.

[5] Elghul M.: Observation on the bacteriophage typing of mycobacterium tuberculosis isolates from the Socialist People's Libyan Arab Jamahiriya. PhD Thesis University of London, London UK 1981.

[6] Awatef A. Ben Ramadan, Mohamed K. Shambesh, Omar I. Abusnena, Mohammad Al-Baccush. Ibnosina: Estimation of the Incidence of Pulmonary Tuberculosis in Northwestern Libya. J Med BS, 2011,3(2):53-58

[7] General Authority of Information of Libya (2008): Results of Census of 2006. pp. 40. The report in Arabic.

[8] World Health Organization (WHO) Global Tuberculosis Control. World Health Organization:Geneva, Switzerland. (2010).

[9] AS et al.: Drug Susceptibility Patterns of Mycobacterium tuberculosis Isolated from Patients with Pulmonary Tuberculosis in Tripoli-Libya, J Mycobac Dis 2014, 4:3 (http://dx.doi.org/10.4172/21611068.1000154)

[10] Issar S: Mycobacterium tuberculosis Pathogenesis and Molecular Determinants of Virulence. J. Clin. Microbiol. (2003). 16:463-496.

[11] World Health Organization Global tuberculosis control: Surveillance, planning, and Financing. (2005). Geneva, World Health Organization.

[12]. EMRO. Regional profile of TB: TB in regional countries. Published by WHO, EMRO, Cairo 2005.

[13] Raja G. H. Abdulrahman, Khola M, H. Abdraba, Atiya F , Mohamed K. Shambesh, Ashraf Albakoush \& Faisal Ismail: Tuberculosis in Tobruk area, Libya: a 30-year: retrospective study: In $1^{\text {st }}$ Medical Research Conference, University of Tripoli. September 2015

[14] Abunaaja S. Lecture notes on biostatistics for medical $\&$ public health students: Demography. The national book store publisher: Benghazi. Libya; 1st edition 2002; 116

[15] EMRO. DOTS in the Mediterranean Region: TB epidemiology in the region. Published by WHO: EMRO, Cairo 2005.

[16] Kadiki O, Ashraf M. Tuberculosis in the Libyan Arab Republic. Published by Ministry of health (Endemic diseases department): Tripoli Libya 1972.

[17]Lords Hansard statement. Immigration: pre-entry screening for tuberculosis. 2010.

[18] Lowenthal P, Westenhouse J, Moore M, Posey DL, Watt JP, et al. Reduced importation of tuberculosis after the implementation of an enhanced pre-immigration screening protocol. Int J Tuberc Lung Dis. (2011) 15: 761-766.

[19] Department of Immigration and Citizenship, Health Policy Section. Instructions for medical and radiological examination of Australia visa applicants. Canberra,
ACT, Australia: Department of Immigration and Citizenship, 2009.

[20] Minister of Public Works and Government Services, Health Management Branch. Handbook for designated medical practitioners. Ottawa, ON,

[21]Canada: Minister of Public Works and Government Services, 2009. 